\title{
Editorial
}

\section{Physicians' Role in Combating Obesity}

\author{
SK Biswas ${ }^{1}, \mathrm{~N} \mathrm{Haque}^{2}$
}

Obesity is defined as a state of abnormal or excessive accumulation of fat in the body that may impair health. Overweight and obesity is rapidly rising and it has reached an epidemic level in many affluent countries. The age-standardized global prevalence of obesity increased from $3.2 \%$ in 1975 to $10.8 \%$ in 2014 in men, and $6.4 \%$ to $14.9 \%$ in women. The prevalence of overweight and obesity in adults has climbed from $7 \%$ in 1980 to $17 \%$ in 2013 in Bangladesh; a rise of more than double. Body mass index (BMI) is an easy and reasonably accurate measure of general obesity or body fatness. BMI is defined or calculated by the weight of a person in kilograms divided by the square of his/her height in meters. The standard BMI cut-off points (BMI 18.5-24.9 kg/m² for normal weight, 25.0-29.9 for overweight, and $\geq 30.0$ for obese persons) are widely accepted all over the world. However, the World Health Organization (WHO) has recommended lowering of BMI cut-off points (BMI 18.5-22.9 $\mathrm{kg} / \mathrm{m}^{2}$ for normal weight, 23.0-27.4 for overweight and $\geq 27.5$ for obese persons) for Asian people considering the increased percentage of body fat and higher risk of cardiovascular and metabolic diseases for Asians compared to European whites of same age, sex and BMI.

The situation of overweight and obesity in Bangladesh must be considered differently compared to many other countries. Bangladesh is a developing country where adequate food security is not yet ensured for all, and more than $20 \%$ men and around $25 \%$ women are still underweight in Bangladesh. The rapidly rising trend of overweight and obesity in Bangladesh where underweight population is still highly prevalent has its unique psychosocial, cultural and economic basis. Due to poverty, ignorance and lack of (health) education lean and thin persons in this society are usually considered by themselves and by others as having ill health. Thus, in this socioeconomic and cultural setting, both normal weight and underweight people have a psychology to gain weight by any means, mostly by overconsumption of processed carbohydrates and other

1. Dr. Subrata Kumar Biswas, MD, PhD, Associate Professor, Department of Biochemistry and Molecular Biology, Bangabandhu Sheikh Mujib Medical University (BSMMU), Dhaka, Bangladesh.

2. Dr. Nasimul Haque, M Phil (Biochemistry) Professor and Head of Department of Biochemistry, Faridpur Medical College, Faridpur, Bangladesh.

Address of correspondence :

Dr. Subrata Kumar Biswas, PhD, Associate Professor, Department of Biochemistry and Molecular Biology, Bangabandhu Sheikh Mujib Medical University (BSMMU), Dhaka, Bangladesh.

Email: su.biswas@yahoo.com unhealthy foods. An extreme example of this tendency is the fact that some people in rural Bangladesh take steroids from quacks to gain weight. At the same time, a large group of affluent people, both educated and uneducated, are also getting fatty since they lead a sedentary life and consume unhealthy foods with no regular exercise. The normal weight and underweight people who gain weight many of them eventually enter within the category of overweight and then become obese. Since the BMI cut-off points have been lowered for Asians, many of those who enter in the category of overweight (BMI 23.0 to $27.4 \mathrm{~kg} / \mathrm{m}^{2}$ ) are normal weight according to standard BMI classification (BMI $<25.0 \mathrm{~kg} / \mathrm{m}^{2}$ ), and many of them are considered as just healthy or in a state of good health rather than overweight. This consideration is very dangerous because some studies have found that overweight and obese subjects have almost similar level of cardiovascular and metabolic risk.

Overweight and obesity directly contribute to the development of cardiovascular diseases, type 2 diabetes mellitus and certain types of cancers. The American Medical Association has recently recognized obesity as a disease considering its disastrous consequences. Thus the physicians have very important role in the early diagnosis and management of obesity. The question is whether the physicians in Bangladesh are ready to fight against the rising trend of overweight and obesity. Unfortunately, majority of the physicians in Bangladesh do not calculate BMI of their patients to diagnose weight status in their practice, and those who do so many of them are not aware of the existence of the WHO recommended lower BMI cut points for the Asians which should be used for Bangladeshi individuals. Furthermore, many ignorant people have a tendency to gain weight due to the misconception that being heavier is healthier. These facts together preclude people from being diagnosed and advised by their physicians at the very early stage of entering into overweight category. Once a person become overweight, he/she finds it very difficult to lose weight and easier to gain more weight. Therefore it may be recommended that our physicians should not ignore overweight status, and should calculate BMI and use Asian BMI criteria to diagnose patients' weight status and advise the patients accordingly. Our policy makers should take necessary measures to educate people about the disastrous consequences of gaining weight. We need a concerted action of common people, health care personnel and policy makers to combat the overweight and obesity-related cardiovascular and metabolic diseases. 